\title{
Heart Stroke Diagnosis using AI Model
}

\author{
Prathamesh D. Mane"1, Dr. Surabhi Thorat ${ }^{2}$ \\ ${ }^{*}$ Masters in Computer Science, Somaiya University, Mumbai, Maharashtra, India \\ ${ }^{2}$ Department of Computer Science, Somaiya University, Mumbai, Maharashtra, India
}

\begin{abstract}
Article Info

Volume 7, Issue 6

Page Number: 241-244

Publication Issue :

November-December-2021

\section{Article History}

Accepted : 02 Dec 2021

Published : 10 Dec 2021

Stroke is a leading cause of disability for adults and the elderly. It can be fatal if it is not treated correctly. Bio- signals can be detected in real-time and can be utilized to improve the treatment of stroke. Most stroke prediction and diagnostic systems rely on image analysis techniques such as computed radiological (CT) or magnetic resonance imaging (MRI). The data cleaning was carried out according to the inclusion criteria that were designed. Two data sets were then created and analyzed using machine learning algorithms. Comparisons of recall rate and accuracy among various algorithms show that the reinforcement learning algorithm is superior to the other two. The experiment utilized three classification algorithms: SVM, linear regression, and Reinforcement learning. The results indicated that Reinforcement Learning is an optimal algorithm for diagnosing complex problems.[1]

Keywords : Support vector machine, International Stroke Trial, Congenital Heart Disease and machine learning algorithms.
\end{abstract}

\section{INTRODUCTION}

Today as increasing in information technology caused in significant growth in science. One of the sciences in medical science. Using artificial intelligence, machine learning, deep learning techniques in all subjects of this branch of science especially cardiovascular diseases made it possible to design medical assistant system.

Cardiovascular diseases of one the most spreading causes of death in worldwide.
What is Stroke?

A stroke, or brain attack, happens when blood flow to your brain is stopped. It is an emergency situation.

The brain needs a constant supply of oxygen and nutrients in order to work well. If blood supply is stopped even for a short time, this can cause problems. Brain cells begin to die after just a few minutes without blood or oxygen.[2]

\section{Kinds of Stroke}

Ischemic stroke - This is the most common type of stroke. It happens when a major blood vessel in the 
brain is blocked. It may be blocked by a blood clot. Or it may be blocked by a buildup of fatty deposit and cholesterol. This buildup is called plaque.[3]

Hemorrhagic stroke - This occurs when a blood vessel in your brain bursts, spilling blood into nearby tissues. With a hemorrhagic stroke, pressure builds up in the nearby brain tissue. This causes even more damage and irritation.[3]

\section{Risk Factors}

1. High Blood Pressure and High Cholesterol

2. Diabetes

3. Smoking and Secondhand Exposure

4. Obesity, Unhealthy Diet and Physical Inactivity

\section{METHODS AND MATERIAL}

1. Data Set Link -

https://www.kaggle.com/rishidamarla/heart-diseaseprediction

\section{Correlation}

The correlation coefficient has values between -1 to 1

- A value closer to 0 implies weaker correlation (exact 0 implying no correlation)

- A value closer to 1 implies stronger positive correlation

- A value closer to -1 implies stronger negative correlation

We after adding dataset to the Project we have to identity the correlation between Features

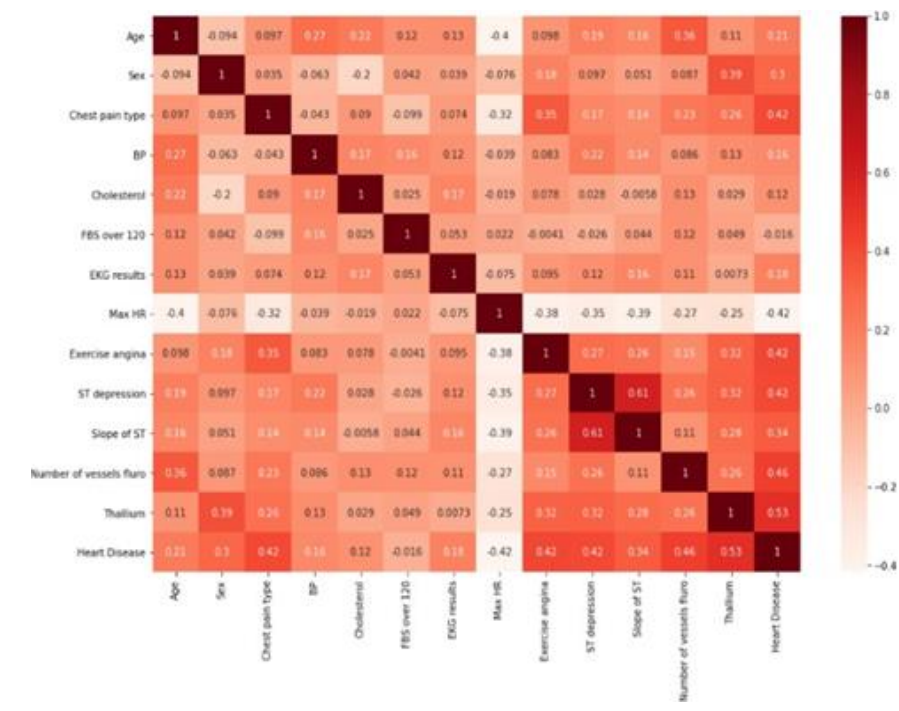

The following Graph shows how the Features are correlated to each other.

\section{Data Preprocessing -}

Data preprocessing is a process of preparing the raw data and making it suitable for a machine learning model. It is the first and crucial step while creating a machine learning model.

I use SelectKBest class to extract top 5 best features and Concat two dataframes for better visualization. After that I use ExtraTreeClassifier model for feature extractction.

\section{ML Algorithm for Classification}

- Linear Regression Model -

- The linear regression approach describes the link between the data and a straight line that can be expressed by the function as accurately as feasible.

- It is possible to express the function of the linear regression model. Since the efficacy of data fitting varies with the kind of the linear model, the model selection is important.

- A function that reflects the difference between the linear regression model and the actual data is used 
Prathamesh D. Mane et al Int. J. Sci. Res. Comput. Sci. Eng. Inf. Technol, November-December-2021, 7 (6) : 241-244

to select a more precise model to describe the

linear connection between data.

\section{KNN Model -}

- K-Nearest Neighbour is one of the simplest Machine Learning algorithms based on Supervised Learning technique.

- $\quad \mathrm{K}-\mathrm{NN}$ algorithm assumes the similarity between the new case/data and available cases and put the new case into the category that is most similar to the available categories.

- K-NN algorithm stores all the available data and classifies a new data point based on the similarity. This means when new data appears then it can be easily classified into a well suite category by using K- NN algorithm.

- $\quad \mathrm{K}-\mathrm{NN}$ algorithm can be used for Regression as well as for Classification but mostly it is used for the Classification problems.

\section{Selecting The best algorithm for Modeling.}

We are going to select best algorithm for our model by comparing results of Linear Regression Model and KNN Algorithms. With the following graph we can say that Linear Regression Model is the best fit for our Dataset.

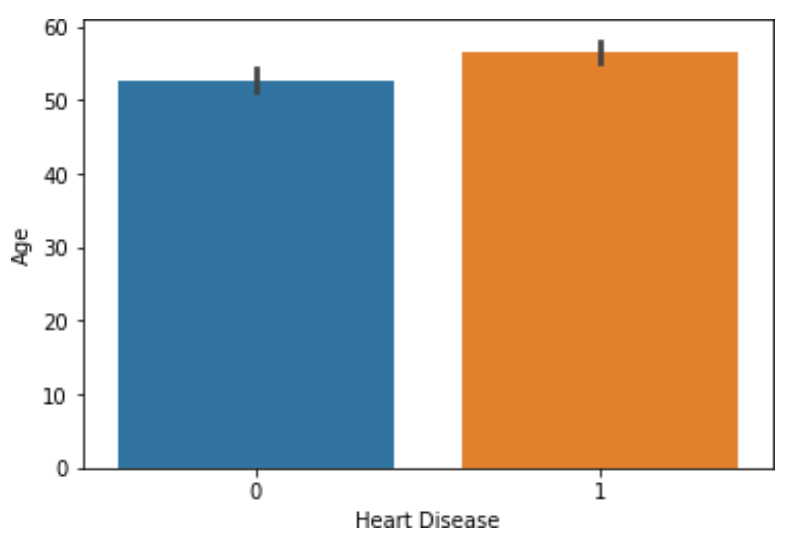

\section{RESULTS AND DISCUSSION}

It should include important findings discussed briefly. Wherever necessary, elaborate on the tables and figures without repeating their contents. Interpret the findings in view of the results obtained in this and in past studies on this topic. State the conclusions in a few sentences at the end of the paper. However, valid colored photographs can also be published.

\section{CONCLUSION}

- Heart is a pump or pulsating pump which composed of four compound holes with two atriums and two ventricles, which delivers blood to all body organs. So the heart is a vital organ of body. Unfortunately todays an important quantity of death is caused by heart diseases. Todays the cardiovascular diseases are the most important challenges of healthcare in the worldwide.

- Prevention and management of cardiovascular diseases requires a pervasive and comprehensive system for recording data. Information of patient records are one of the most important data, which must be classified for easy and fast treatment process. Goal of current study was providing a classifying system for cardiovascular diseases to improve the policies of healthcare in opposition against cardiovascular diseases.

- The main goal in classifying is putting people in groups with predetermined number of patients. Then the future patients will be helped with evaluating their signs.

- In all developed countries the diseases classifying system is a fundamental base for addressing healthcare requirements of country with classifying cardiovascular diseases.

- The national system of classifying cardiovascular diseases will play an effective role in improving the management and prevention of cardiovascular diseases in Iran. Using this 
approach we can greatly help in early prediction of this disease.

\section{REFERENCES}

[1]. G. Sasikala, G. Roja and D. Radhika "Prediction Of Heart Stroke Diseases Using Machine Learning Technique Based Electromyographic Data", 2021 June; Vol.12 No 13 (2021), 44244431.

[2]. Silvia Romiti, Mattia Vinciguerra, Wael Saade, Iñaki Anso Cortajarena, and Ernesto Greco. Artificial Intelligence (AI) and Cardiovascular Diseases: An Unexpected Alliance. 2020 June 27 ;4972346.

[3]. Jaehak Yu, Sejin Park, Soon-Hyun Kwon, Chee Meng Benjamin Ho, Cheol-Sig Pyo and Hansung Lee. AI- Based Stroke Disease Prediction System Using Real- Time Electromyography Signals 2020 Oct 6791.

\section{Cite this article as :}

Prathamesh D. Mane, Dr. Surabhi Thorat, "Heart Stroke Diagnosis using AI Model", International Journal of Scientific Research in Computer Science, Engineering and Information Technology (IJSRCSEIT), ISSN : 2456-3307, Volume 7 Issue 6, pp. 241-244, November-December 2021. Available at doi : https://doi.org/10.32628/CSEIT217652

Journal URL : https://ijsrcseit.com/CSEIT217652 